\title{
Observer synthesis under time-varying sampling for Lipschitz nonlinear systems
}

\author{
Lucien Etienne $^{\mathrm{a}}$, Laurentiu Hetel ${ }^{\mathrm{b}}$, Denis Efimov ${ }^{\mathrm{a}}$, Mihaly Petreczky ${ }^{\mathrm{b}}$ \\ ${ }^{a}$ Non-A team at Inria Lille, Parc Scientifique de la Haute Borne, 40 avenue Halley, 59650 Villeneuve d'Ascq, France, \\ (e-mail: lucien.etienne@inria.fr,denis.efimov@inria.fr) \\ ${ }^{\mathrm{b}}$ Centre de Recherche en Informatique, Signal et Automatique de Lille (CRIStAL, CNRS UMR 9189), Ecole Centrale de \\ Lille, 59650 Villeneuve d'Ascq, France, (e-mail: laurentiu.hetel@ec-lille.fr, mihaly.petreczky@ec-lille.fr)
}

\begin{abstract}
In this work, the problem of observation of continuous-time nonlinear Lipschitz systems under time-varying discrete measurements is considered. This class of systems naturally occurs when continuous processes are observed through digital sensors and information is sent via a network to a computer for state estimation. Since the network introduces variations in the sampling time, the observer must be designed so to take them into account. Here impulsive observers, which make instantaneous correction when information is received, are investigated. Moreover, we consider time-varying observer gains adapting to the varying sampling interval. In order to deal with both continuous-time and discrete-time dynamics, a new hybrid model is used to state the problem and establish the convergence of the proposed observer. First, generic conditions are provided using a hybrid Lyapunov function. Then a restriction of the generic Lyapunov function is used to establish tractable conditions that allows the synthesis of an impulsive gain.
\end{abstract}

Key words: Impulsive observer, Continuous-discrete observers, Nonlinear systems, Hybrid systems.

\section{Introduction}

Observation for linear and nonlinear systems constitutes a large area of study, which is primarily motivated by the fact that measuring all variables of interest of a system may be infeasible. The sensing of a continuous process is usually done digitally and information is sent through networks to a computer for state estimation. Due to communication constraints introduced by the network, the sensor may not be able to communicate periodically [9], [11], [13], [19]. Thus sampling jitter are introduced in the control loop. This jitter can degrade the performance of the system and they can even be a source of instability $[12,14]$. In this context, an observer should be robust with respect to time varying sampling. The aim of this work is to design an observer for continuous-time Lipschitz nonlinear systems where measurements are made discretely and with aperiodic sampling instants.

In recent years the problem of observer synthesis for Lipschitz systems with discrete measurements has been the subject of different studies. See [1],[16] for systems with periodic sampling and [3],[4],[5],[15],[17] for systems considering time-varying sampling intervals. From a methodological point of view, [3] and [16] are based on the use of impulsive models and time-varying Lyapunov functions. The work in [5] is based on a hight gain observer approach. The observer scheme uses the last sampled measurement to continuously correct the observation error state. A notable difference with respect to the literature is the use of observers with time-varying gains: the gain of the

\footnotetext{
1 This work has received funding from Region Nord-Pas de Calais through theARCIR project ESTIREZ, the ANR through the JC-JC project ROCC-SYS (agreementANR-14-CE27-0008)and the European Union's Horizon2020 research and innovation programme under the Marie Sklodowska-Curie grant agreement No 675080(UCoCoS). The material in this paper was not presented at any conference.
} 
observer exponentially decreases as the time from the last sampled measurement increases. In order to avoid some conservative over-approximations due to nonlinearities, [1], [4], [15] propose a new method based on the computation of reachable sets of the observation error between sampling instants. This method involves the offline computation of convex polytopes embedding the transition matrix in between sampling instants and the use of discrete-time Lyapunov conditions. In the same spirit, the work in [6] considers the case of linear systems. Similarly to [1], [4], [15], the authors of [6] use a convex embedding of the transition matrix. However, the inter-sampling system behaviour is captured using a hybrid framework [10].

Considering the problem of observation for nonlinear Lipschitz system with discrete, time-varying measurements, here we propose an alternative approach. The method is based on a new hybrid model describing the observation error and it suggests the use of new classes of Lyapunov functions. This method allows to take advantage of the best features of the existing approaches. We study the existence of impulsive observers with time-varying gains depending on the inter-sampling time. New generic stability conditions are presented in the form of parametric LMIs. The proposed conditions avoid the use of complex computations of convex embeddings of the system transition matrix. They lead to new theoretic criteria for observer synthesis. Furthermore, we show how to derive simple numerically tractable conditions from the proposed theoretical ones. A finite set of LMIs is given allowing for the computation of (time-varying) observer gains while ensuring asymptotic stability of the observation error. The observation with static gain is recovered as a special instance of our more general result.

This paper is structured as follows. In section 2 the model of the plant and the impulsive observer are given, then some notion of hybrid systems are recalled, and the system under study is restated as a hybrid system. In section 3 we state our main results. General conditions for observer synthesis along with numerically solvable conditions are given. Last in section 4, we illustrate our approach on the model of a flexible joint.

Notation: $v^{\prime}$ denote the transpose of $v$ for either a matrix or a vector. For a symmetric matrix the symbol $\star$ denotes the elements induced by symmetry: $\left(\begin{array}{cc}A & B \\ B^{\prime} & C\end{array}\right)$ will be denoted $\left(\begin{array}{cc}A & B \\ \star & C\end{array}\right)$. Given a matrix $A$, we introduce the notation $\mathfrak{H} \mathfrak{e}(A)=A+A^{\prime}$. The number of elements of a set $\mathcal{P}$ will be denoted by $\operatorname{Card}(\mathcal{P})$. $\mathbb{R}_{\geq 0}$ corresponds to the positive real numbers. Given $p \in \mathbb{N}, \Delta_{p}$ denotes the unit simplex,

$$
\Delta_{p}:=\left\{\delta \in \mathbb{R}_{\geq 0}^{p}: \sum_{i=1}^{p} \delta_{i}=1\right\}
$$

For a set of matrices $R_{i} \in \mathbb{R}^{n \times m}, i=1, \ldots p, \operatorname{Cov}\left\{R_{i}\right\}_{i \in\{1 \ldots p\}}$ denotes its closed convex hull

$$
\operatorname{Cov}\left\{R_{i}\right\}_{i \in\{1 \ldots p\}}=\left\{Z: \exists \delta \in \Delta_{p}, \sum_{i=1}^{p} \delta_{i} R_{i}=Z\right\}
$$

The euclidean norm of $x \in \mathbb{R}^{n}$ is denoted by $|x|$. The distance from a point $x \in \mathbb{R}^{n}$ to a closed set $\mathcal{A} \subset \mathbb{R}^{n}$ is defined as $|x|_{\mathcal{A}}:=\inf _{y \in \mathcal{A}}|x-y|$. In the sequel, $\lambda_{\max }(Q)$ (resp. $\lambda_{\min }(Q)$ ) denotes the biggest (resp. smallest) eigenvalue of a symmetric matrix $Q$. For a square matrix $P>0$ (resp. $P<0$ ) means that $P$ is positive definite (resp. negative definite). $\alpha: \mathbb{R}_{\geq 0} \rightarrow \mathbb{R}_{\geq 0}$ is a class $\mathcal{K}$ function (written $\alpha \in \mathcal{K}$ ) if $\alpha$ is continuous, strictly increasing and $\alpha(0)=0$, if furthermore $\alpha$ is unbounded, $\alpha$ is said to be $\mathcal{K}_{\infty}\left(\alpha \in \mathcal{K}_{\infty}\right)$. A function $\rho: \mathbb{R}_{>0} \rightarrow \mathbb{R}_{>0}$ is a $\mathcal{P D}$ function if $\forall s>0, \rho(s)>0$ and $\rho(0)=0$. For a given left continuous function $w$, we write the left-hand limit at the point $s$ as $w\left(s^{-}\right)=\lim _{t<s, t \rightarrow s} w(t)$.

\section{Problem statement}

\subsection{System description}

We consider a nonlinear Lipschitz system of the form

$$
\begin{aligned}
& \dot{x}(t)=A x(t)+B u(t)+G \phi(H x(t)), t \geq 0, \\
& x(0)=x_{0} \in \mathbb{R}^{n}, \\
& y_{k}=C x\left(t_{k}\right), k \in \mathbb{N},
\end{aligned}
$$


with $x \in \mathbb{R}^{n}$ the state and $u \in \mathbb{R}^{l}$ the input applied to the system. Here $A \in \mathbb{R}^{n \times n}, B \in \mathbb{R}^{n \times l}, C \in \mathbb{R}^{q \times n}, G \in \mathbb{R}^{n \times m}$ and $H \in \mathbb{R}^{m \times n}$ are known matrices. The nonlinear term $\phi: \mathbb{R}^{m} \rightarrow \mathbb{R}^{m}$ satisfies the Lipschitz condition:

$$
|\phi(a)-\phi(b)| \leq \gamma|a-b|, \quad \forall(a, b) \in \mathbb{R}^{m} \times \mathbb{R}^{m},
$$

for some $\gamma>0$. Furthermore, it is assumed that $u(t)$ is piecewise continuous and bounded. We denote by $\left(t_{k}\right)_{k \in \mathbb{N}}$ the sequence of sampling times. We assume that $t_{0}=0$ and that $\left(t_{k}\right)_{k \in \mathbb{N}}$ is monotonously increasing. $y_{k} \in \mathbb{R}^{p}$ represents the sampled output available at time $t_{k}$. In what follows, it is assumed that there exist both a minimal $(\underline{\tau}>0)$ and a maximal time $(\bar{\tau} \geq \underline{\tau})$ between two consecutive sampling instants. Therefore we can define the set of all admissible sampling sequences by:

$$
S_{[\underline{\tau}, \bar{\tau}]}:=\left\{\left(t_{k}\right)_{k \in \mathbb{N}}: t_{0}=0, t_{k+1}-t_{k} \in[\underline{\tau}, \bar{\tau}], k \in \mathbb{N}\right\} .
$$

It follows from the equations of system (1) and the assumption in (2) that for every initial condition $x_{0} \in \mathbb{R}^{n}$ and every time $t \geq 0$ the solution of system (1) exists and is unique [8]. For the system under study we introduce an impulsive observer

$$
\begin{gathered}
\dot{\hat{x}}(t)=A \hat{x}(t)+B u(t)+G \phi(H \hat{x}(t)), \\
t \in\left[t_{k}, t_{k+1}\right), \\
\hat{x}\left(t_{k}\right)=\hat{x}\left(t_{k}^{-}\right)+K\left(t_{k}-t_{k-1}\right) C\left(x\left(t_{k}^{-}\right)-\hat{x}\left(t_{k}^{-}\right)\right) \\
k \in \mathbb{N}, \quad k \geq 1, \\
\hat{x}(0)=\hat{x}_{0} \in \mathbb{R}^{n},
\end{gathered}
$$

where $\hat{x} \in \mathbb{R}^{n}$ represents the state of the observer and $K:[\underline{\tau}, \bar{\tau}] \rightarrow \mathbb{R}^{n \times q}$ a continuous and bounded time-varying gain. Between sampling instants the observer is a copy of the system. The state of the observer is reset using the new available measurement when information is received. The goal of the article is to design the gain function $K($. so as to ensure the convergence of the observer (3) to the state of system (1).

Denoting the observation error $z=x-\hat{x}$, we obtain the following dynamics for the observation error:

$$
\begin{aligned}
& \dot{z}(t)=A z(t)+G[\phi(H x(t))-\phi(H(x(t)-z(t)))], \\
& t \in\left[t_{k}, t_{k+1}\right), \\
& z\left(t_{k}\right)=\left(I-K\left(t_{k}-t_{k-1}\right) C\right) z\left(t_{k}^{-}\right), \\
& k \in \mathbb{N}, k \geq 1 \text {, }
\end{aligned}
$$

The solutions of both (3) and (4) are defined in an iterative way. For all $t \in\left[t_{k}, t_{k+1}\right)$ the solution is defined by $(3 \mathrm{a}),(4 \mathrm{a})$. Since (1) is Lipschitz, the existence and uniqueness of $\hat{x}(t)$ (resp. $z(t)$ ) on $\left[t_{k}, t_{k+1}\right)$ is guaranteed for a fixed $\hat{x}\left(t_{k}\right)$ (resp. $z\left(t_{k}\right)$. At time $t_{k}$, using the left limits $\hat{x}\left(t_{k}^{-}\right)\left(\right.$resp. $\left.z\left(t_{k}^{-}\right)\right)$along with $(3 \mathrm{~b}),(4 \mathrm{~b}), \hat{x}\left(t_{k}\right)\left(\right.$ resp. $\left.z\left(t_{k}\right)\right)$ gives the initial condition of the differential equation (3a) (resp. (4a)) for $\left[t_{k}, t_{k+1}\right)$.

\subsection{Generalities on hybrid systems}

Next, we will rewrite (4) into the hybrid framework described in [10]. Consider a hybrid system

$$
\mathcal{H}: \begin{cases}\xi \in C_{\mathcal{H}}, & \dot{\xi} \in F_{\mathcal{H}}(\xi), \\ \xi \in D_{\mathcal{H}}, & \xi^{+} \in G_{\mathcal{H}}(\xi) .\end{cases}
$$

Roughly speaking, while $\xi$ belongs to $C_{\mathcal{H}}$, the state flows according to a differential inclusion characterized by a set-valued mapping $F_{\mathcal{H}}$. When $\xi$ belongs to $D_{\mathcal{H}}$, the state jumps according to a discrete dynamic defined by $G_{\mathcal{H}}$. In what follows we will use the concepts and notations from [10]. The most important are recalled below.

Definition 1 (Domain of a set-valued mapping) Given a set-valued mapping $M: \mathbb{R}^{m} \rightrightarrows \mathbb{R}^{n_{\xi}}$, the domain of $M$ is the set $\operatorname{dom} M=\left\{x \in \mathbb{R}^{m}: M(x) \neq \emptyset\right\}$. 
Definition 2 (Data of a hybrid system) The data of a hybrid system $\mathcal{H}$ in $\mathbb{R}^{n_{\xi}}$ consists of four elements:

- $a$ set $C_{\mathcal{H}} \subset \mathbb{R}^{n_{\xi}}$, called the flow set;

- a set-valued mapping $F_{\mathcal{H}}: \mathbb{R}^{n_{\xi}} \rightrightarrows \mathbb{R}^{n_{\xi}}$ with $C_{\mathcal{H}} \subset$ dom $F_{\mathcal{H}}$, called the flow map;

- a set $D_{\mathcal{H}} \subset \mathbb{R}^{n_{\xi}}$, called the jump set;

- a set-valued mapping $G_{\mathcal{H}}: \mathbb{R}^{n_{\xi}} \rightrightarrows \mathbb{R}^{n_{\xi}}$ with $D_{\mathcal{H}} \subset$ dom $G_{\mathcal{H}}$, called the jump map.

Definition 3 (Hybrid time domains) A subset $E \subset \mathbb{R}_{\geq 0} \times \mathbb{N}$ is a compact hybrid time domain if

$$
E=\cup_{j=0}^{J-1}\left(\left[t_{j}, t_{j+1}\right], j\right)
$$

for some finite sequence of times $0=t_{0} \leq t_{1} \leq t_{2} \leq \ldots \leq t_{J}$. It is a hybrid time domain if for all $(T, J) \in$ $E, E \cap([0, T] \times\{0,1, \ldots, J\})$ is a compact hybrid domain.

Definition 4 (Hybrid arc) A function $\xi: E \rightarrow \mathbb{R}^{n_{\xi}}$ is a hybrid arc if $E$ is a hybrid time domain and if for each $j \in \mathbb{N}$, the function $t \rightarrow \xi(t, j)$ is locally absolutely continuous ${ }^{2}$ on the interval $I_{j}=\{t:(t, j) \in E\}$.

Definition 5 (Solution to a hybrid system) A hybrid arc $\xi$ is a solution to the hybrid system $\mathcal{H}$ if $\xi(0,0) \in$ $C_{\mathcal{H}} \cup D_{\mathcal{H}}$, and

$\left(S_{1}\right)$ for all $j \in \mathbb{N}$ such that $I_{j}:=\{t:(t, j) \in$ dom $\xi\}$ has nonempty interior,

$$
\begin{gathered}
\xi(t, j) \in C_{\mathcal{H}} \text { for all } t \in \operatorname{int}\left(I_{j}\right), \\
\dot{\xi}(t, j) \in F_{\mathcal{H}}(\xi(t, j)) \text { for almost all } t \in I_{j}
\end{gathered}
$$

$\left(S_{2}\right)$ for all $(t, j) \in \operatorname{dom} \xi$ such that $(t, j+1) \in \operatorname{dom} \xi$

$$
\xi(t, j) \in D_{\mathcal{H}}, \xi(t, j+1) \in G_{\mathcal{H}}(\xi(t, j))
$$

Definition 6 (Maximal solutions) A solution $\xi$ to $\mathcal{H}$ is maximal if there does not exist another solution $\psi$ to $\mathcal{H}$ such that dom $\xi$ is a proper subset of dom $\psi$ and $\xi(t, j)=\psi(t, j)$ for all $(t, j) \in$ dom $\xi$.

A solution is called complete if it is maximal and defined on an unbounded hybrid time domain. We define as follows the concept of Uniform Global pre-Asymptotic stability (UGpAS) that will be used in the rest of the article.

Definition 7 (UGpAS) Consider a hybrid system $\mathcal{H}$ on $\mathbb{R}^{n_{\xi}}$. Let $\mathcal{A} \subset \mathbb{R}^{n_{\xi}}$ be closed. The set $\mathcal{A}$ is said to be

- uniformly globally stable for $\mathcal{H}$ if there exists a class- $\mathcal{K}_{\infty}$ function $\alpha$ such that any solution $\xi$ to $\mathcal{H}$ satisfies $|\xi(t, j)|_{\mathcal{A}} \leq \alpha\left(|\xi(0,0)|_{\mathcal{A}}\right)$ for all $(t, j) \in \operatorname{dom} \xi$

- uniformly globally pre-attractive for $\mathcal{H}$ if for each $\varepsilon>0$ and $r>0$ there exists $T>0$ such that, for any solution $\xi$ to $\mathcal{H}$ with $|\xi(0,0)|_{\mathcal{A}} \leq r,(t, j) \in \operatorname{dom} \xi$ and $t+j \geq T$ imply $|\xi(t, j)|_{\mathcal{A}} \leq \varepsilon$

- uniformly globally pre-asymptotically stable (UGpAS) for $\mathcal{H}$ if it is both uniformly globally stable and uniformly globally pre-attractive.

If furthermore all the maximal solutions of (5) are complete then we say that $\mathcal{A}$ is Uniformly Globally Asymptotically stable (UGAS)

Definition 8 (Candidate Lyapunov function ) A function $V: \operatorname{dom} V \rightarrow \mathbb{R}$ is said to be a candidate Lyapunov function for the hybrid system $\mathcal{H}$ if the following conditions hold:

1. $\overline{C_{\mathcal{H}}} \cup D_{\mathcal{H}} \cup G_{\mathcal{H}}\left(D_{\mathcal{H}}\right) \subset \operatorname{dom} V$;

2. $V$ is continuously differentiable on an open set containing $\overline{C_{\mathcal{H}}}$, where $\overline{C_{\mathcal{H}}}$ denotes the closure of $C_{\mathcal{H}}$.

We recall now the Theorem 3.18 of [10]:

\footnotetext{
$\overline{2}$ Local absolute continuity means that $t \rightarrow \xi(t, j)$ is differentiable almost everywhere on each $I_{j}$ with non empty interiors see for example [10] p. 28.
} 
Theorem 1 (Sufficient conditions for UGpAS) Let $\mathcal{H}$ be a hybrid system and let $\mathcal{A} \subset \mathbb{R}^{n}$ be closed. If $V$ is a Lyapunov function candidate for $\mathcal{H}$ and there exist $\alpha_{1}, \alpha_{2} \in \mathcal{K}_{\infty}$, and a continuous $\rho \in \mathcal{P} \mathcal{D}$ such that:

$$
\begin{gathered}
\forall \xi \in C_{\mathcal{H}} \cup D_{\mathcal{H}} \cup G_{\mathcal{H}}\left(D_{\mathcal{H}}\right) \\
\alpha_{1}\left(|\xi|_{\mathcal{A}}\right) \leq V(\xi) \leq \alpha_{2}\left(|\xi|_{\mathcal{A}}\right) \\
\forall \xi \in C_{\mathcal{H}}, f \in F_{\mathcal{H}}(\xi),\langle\nabla V(\xi), f\rangle \leq-\rho\left(|\xi|_{\mathcal{A}}\right) \\
\forall \xi \in D_{\mathcal{H}}, g \in G_{\mathcal{H}}(\xi), V(g)-V(\xi) \leq-\rho\left(|\xi|_{\mathcal{A}}\right)
\end{gathered}
$$

Then $\mathcal{A}$ is UGpAs for $\mathcal{H}$.

\subsection{Hybrid reformulation}

Next, we will reformulate (4) into a hybrid framework. We use the fact that the dynamics of (4a) can be embedded in a polytopic set of matrices. This method is well-known in the literature and can also be applied to some classes of non Lipschitz system. See for instance the work of [20]. In our setting it can be stated as the following:

Lemma 1 [20] Consider equations (1),(3),(4). Then, there exists a finite set of matrices $R_{i}, i \in \mathcal{P}:=\{1,2, \ldots, p\}$, such that for any $(x, z) \in \mathbb{R}^{n} \times \mathbb{R}^{n}$

$$
A z+G[\phi(H x)-\phi(H(x-z))] \in \operatorname{Cov}\left\{R_{i} z\right\}_{i \in \mathcal{P}} .
$$

In practice, the set of matrices $R_{i}, i \in \mathcal{P}$ can be easily computed numerically. See [20] for further details.

Using Lemma 1, system (4) can be described by:

$$
\left.\begin{array}{l}
\dot{z} \in \operatorname{Cov}\left\{R_{i} z\right\}_{i \in \mathcal{P}} \\
\dot{\tau}=1 \\
\dot{s}=-1
\end{array}\right\}(\tau, s) \in[0, \bar{\tau}]^{2},
$$

and

$$
\left.\begin{array}{rl}
z^{+} & =(I-K(\tau) C) z \\
\tau^{+} & =0 \\
s^{+} & \in[\underline{\tau}, \bar{\tau}]
\end{array}\right\}(s=0) \wedge(\tau \in[\underline{\tau}, \bar{\tau}]) .
$$

Here (7) corresponds to the system dynamic in between sampling times, i.e. to (4a), while (8) describes the impulsive dynamics $(4 \mathrm{~b})$. The system state is augmented to include two clocks. A decreasing one, $s \in[0, \bar{\tau}]$, giving the time before the next sampling occurs, and an increasing one, $\tau \in[0, \bar{\tau}]$ which accounts for the time elapsed since the last sampling. The set-valued mapping reset of $s$ in (8) captures the aperiodic nature of the sampling sequences in $\mathcal{S}_{[\tau, \bar{\tau}]}$. Depending on application the value of $s$ may be known or unknown. However, the value of $\tau$ can be measured and used as an argument of $K(\cdot)$.

Defining the extended state $\xi:=\left(z^{\prime}, \tau, s\right)^{\prime}$, the hybrid system (7),(8) can be rewritten in the form (5) with

$$
C_{\mathcal{H}}=\left\{\xi \in \mathbb{R}^{n+2} \mid z \in \mathbb{R}^{n},(\tau, s) \in[0, \bar{\tau}]^{2}\right\}
$$

the flow map

$$
F_{\mathcal{H}}(\xi)=\left(\begin{array}{c}
\operatorname{Cov}\left\{R_{i} z\right\}_{i \in \mathcal{P}} \\
1 \\
-1
\end{array}\right)
$$


for $\xi$ in $C_{\mathcal{H}}$ and $F_{\mathcal{H}}(\xi)=\emptyset$ elsewhere; the jump set

$$
D_{\mathcal{H}}=\left\{\xi \in \mathbb{R}^{n+2} \mid, z \in \mathbb{R}^{n},(\tau \in[\underline{\tau}, \bar{\tau}]) \wedge(s=0)\right\}
$$

and the jump map

$$
G_{\mathcal{H}}(\xi)=\left(\begin{array}{c}
(I-K(\tau) C) z \\
0 \\
{[\underline{\tau}, \bar{\tau}]}
\end{array}\right)
$$

when $\xi$ in $D_{\mathcal{H}}$ and $G_{\mathcal{H}}(\xi)=\emptyset$ elsewhere.

Remark 1 Consider a solution $z$ of system (4) with $x$ satisfying (1) and $\left(t_{k}\right)_{k \in \mathbb{N}} \in S_{[\tau, \bar{\tau}]}$. Given $E=\cup_{k=0}^{\infty}\left(\left[t_{k}, t_{k+1}\right], k\right)$, define the hybrid arc $\xi: E \rightarrow \mathbb{R}^{n+2}$ as follows

$$
\xi(t, k)=\left(z^{\prime}(t), t-t_{k}, t_{k+1}-t\right)^{\prime}, t \in\left[t_{k}, t_{k+1}\right)
$$

and

$$
\xi\left(t_{k+1}, k\right)=\lim _{t \rightarrow t_{k+1}, t<t_{k+1}}\left(z^{\prime}(t), t-t_{k}, t_{k+1}-t\right)^{\prime}
$$

Using Lemma 1 and the description (7),(8), one can see that $\xi$ is a complete solution to the hybrid system (5) with $\operatorname{data} C_{\mathcal{H}}, F_{\mathcal{H}}, D_{\mathcal{H}}, G_{\mathcal{H}}$ as in (9)-(12).

For system (5), (9)-(12) we consider UGpAS with respect to the set

$$
\mathcal{A}=\left\{\xi \in C_{\mathcal{H}} \cup D_{\mathcal{H}} \mid z=0, \tau \in[0, \bar{\tau}], s \in[0, \bar{\tau}]\right\}
$$

The problem addressed here is formalized as follows:

Problem: Consider system (5), (9)-(12). Design the gain function $K(\tau)$ such that the set $\mathcal{A}$ is UGpAS.

Remark 2 The UGpAS of the set $\mathcal{A}$ for the hybrid system (5), (9)-(12) implies that the equilibrium $z=0$ of the observation error (4) is asymptotically stable. Note that for any $\xi \in C_{\mathcal{H}} \cup D_{\mathcal{H}},|\xi|_{\mathcal{A}}=|z|$. From Remark 1 , UGpAS for the set $\mathcal{A}$ defined in (15) for (5), (9)-(12) implies for the solutions $z$ of system (4) that there exist a $\mathcal{K}_{\infty}$ function $\alpha$ such that

$$
|z(t)| \leq \alpha(|z(0)|), \forall t \geq 0
$$

Furthermore, for each $\varepsilon>0$ and $r>0$ there exists $\tilde{T}>0$ such that, if $|z(0)| \leq r$, and $t \geq \tilde{T}$ then $|z(t)| \leq \varepsilon$. This implies that $\lim _{t \rightarrow \infty} z(t)=0$.

\section{Main Results}

\subsection{Time dependent impulsive gain}

First we provide UGpAS analyzis conditions for a given time-varying observer gain. The proposed conditions are based on the existence of a Lyapunov function of the form $V(\xi)=z^{\prime} P(\tau, s) z$ satisfying the assumptions given by Definition 8 and Theorem 1 .

Theorem 2 Consider the hybrid system (5), (9)-(12), a matrix function $P(\tau, s), P: \mathbb{R} \times \mathbb{R} \rightarrow \mathbb{R}^{n \times n}$, with $\frac{\partial P}{\partial \tau}, \frac{\partial P}{\partial s}$ 
being continuous on an open set containing $[0, \bar{\tau}]^{2}$, and the following set of LMIs

$$
\begin{aligned}
& P(\tau, s)=P(\tau, s)^{\prime}>0, \forall(\tau, s) \in[0, \bar{\tau}]^{2}, \\
& N_{i}(\tau, s)=\mathfrak{H} \mathfrak{e}\left(P(\tau, s) R_{i}\right)+\frac{\partial P}{\partial \tau}-\frac{\partial P}{\partial s}<0, \\
& \forall(\tau, s) \in[0, \bar{\tau}]^{2}, \forall i \in \mathcal{P}, \\
& \left(\begin{array}{c|c}
P(0, s) K(\tau) C & C^{\prime} K(\tau)^{\prime} P(0, s) \\
+P(\tau, 0)-P(0, s) & \\
\star \star & P(0, s)
\end{array}\right)>0, \\
& \forall(\tau, s) \in[\underline{\tau}, \bar{\tau}]^{2} .
\end{aligned}
$$

If the LMIs (16) are verified, then the set $\mathcal{A}$ defined in (15) is UGpAS.

PROOF. Here, we show that the set $\mathcal{A}$ is UGpAS using the Theorem 1 . Define $V(\xi)=z^{\prime} P(\tau, s) z$. First $V$ is a candidate hybrid Lyapunov function in the sense of Definition 8 since $\overline{C_{\mathcal{H}}} \cup D_{\mathcal{H}} \cup G_{\mathcal{H}}\left(D_{\mathcal{H}}\right) \subset \operatorname{dom} V$ and $V$ is continuously differentiable on an open set containing $\overline{C_{\mathcal{H}}}$.

One has $\forall \xi \in C_{\mathcal{H}} \cup D_{\mathcal{H}} \cup G_{\mathcal{H}}\left(D_{\mathcal{H}}\right)=C_{\mathcal{H}}$,

$$
\lambda_{\min }|z|^{2}=\lambda_{\min }|\xi|_{\mathcal{A}}^{2} \leq V(\xi) \leq \lambda_{\max }|\xi|_{\mathcal{A}}^{2}=\lambda_{\max }|z|^{2},
$$

where $\lambda_{\min }:=\min _{(\tau, s) \in[0, \bar{\tau}]^{2}}\left\{\lambda_{\min }(P(\tau, s))\right\}$ and $\lambda_{\max }:=\max _{(\tau, s) \in[0, \bar{\tau}]^{2}}\left\{\lambda_{\max }(P(\tau, s))\right\}$. Therefore (6a) is satisfied.

Conditions during flow $\left(\xi \in C_{\mathcal{H}}\right)$ :

Note that $\forall \xi \in C_{\mathcal{H}}, \forall f \in \mathcal{F}_{\mathcal{H}}(\xi)$

$$
\langle\nabla V, f\rangle=\left(\frac{\partial V}{\partial z}, \frac{\partial V}{\partial \tau}, \frac{\partial V}{\partial s}\right)\left(\begin{array}{c}
\sum_{i \in \mathcal{P}} \lambda_{i} R_{i} z \\
1 \\
-1
\end{array}\right),
$$

for some $\lambda \in \Delta_{p}$. Writing $R(\lambda)=\sum_{i \in \mathcal{P}} \lambda_{i} R_{i}$, one has

$$
\begin{array}{r}
\langle\nabla V, f\rangle=z^{\prime} P(\tau, s) R(\lambda) z+z^{\prime} R(\lambda)^{\prime} P(\tau, s) z+ \\
z^{\prime} \frac{\partial P(\tau, s)}{\partial \tau} z-z^{\prime} \frac{\partial P(\tau, s)}{\partial s} z .
\end{array}
$$

which can be expressed as $\langle\nabla V, f\rangle=\sum_{i \in \mathcal{P}} \lambda_{i} z^{\prime} N_{i}(\tau, s) z$ with $N_{i}(\tau, s)$ given in (16b). Since the LMIs (16b) are strict, there exists a sufficiently small $\varepsilon>0$ such that

$$
\forall i \in \mathcal{P}, \forall(\tau, s) \in[0, \bar{\tau}]^{2}, N_{i}(\tau, s)<-\varepsilon I .
$$

Thus $\forall \xi \in C_{\mathcal{H}}, \forall f \in \mathcal{F}_{\mathcal{H}}(\xi)$,

$$
\langle\nabla V, f\rangle=z^{\prime}\left(\sum_{i \in \mathcal{P}} \lambda_{i} N_{i}(\tau, s)\right) z \leq-\varepsilon z^{\prime} z=-\varepsilon|\xi|_{\mathcal{A}}^{2}
$$

that is $(6 \mathrm{~b})$ is verified.

Conditions during jump $\left(\xi \in D_{\mathcal{H}}\right)$ :

From equation (16c), applying the Shur complement it follows that,

$$
(I-K(\tau) C)^{\prime} P(0, s)(I-K(\tau) C)-P(\tau, 0)<0
$$


Since the previous LMIs are strict, there exists a sufficiently small $\tilde{\varepsilon}>0$ such that

$$
\begin{aligned}
& \forall(\tau, s) \in[\underline{\tau}, \bar{\tau}]^{2} \\
& z^{\prime}(I-K(\tau) C)^{\prime} P(0, s)(I-K(\tau) C) z- \\
& z^{\prime} P(\tau, 0) z \leq-\tilde{\varepsilon} z^{\prime} z .
\end{aligned}
$$

Using the evolution of the system at jumping time (8),

$$
V(g)-V(\xi) \leq-\varepsilon^{\prime}|\xi|_{\mathcal{A}}^{2}, \forall \xi \in D_{\mathcal{H}}, \forall g \in G_{\mathcal{H}}\left(D_{\mathcal{H}}\right) .
$$

All the conditions of Theorem 1 are verified. The set $\mathcal{A}$ is UGpAS.

Remark 3 The previous theorem provides sufficient conditions for the UGpAs of set $\mathcal{A}$ in (15) associated to the hybrid system (5), (9)-(12). In view of Remark 2, the proposed conditions also imply asymptotic stability for the trivial equilibrium $z=0$ of the observation error (4). In what follows we show how they can be used for observer design.

Remark 4 We would like to make a link with other approaches focusing on a time delay point of view for the stability analysis of sampled-data system. In our modelling framework, we include both an increasing clock $\tau$ and a decreasing one $s$. Then, the candidate Lyapunov function $V(\xi)=z^{T} P(\tau, s) z$ recaptures the structure of previously developed Lyapunov-Krasovskii functional [7] for LTI systems. Consider equation (1) with $\phi(H x)=0$. The LyapunovKrasovskii functional proposed in [7] has the form:

$$
\begin{aligned}
\bar{V}(t)= & z^{\prime}(t) \bar{P} z(t)+\left(t_{k+1}-t\right) \int_{t_{k}}^{t} \dot{z}^{\prime}(\theta) U \dot{z}(\theta) d \theta \\
& +\left(t_{k+1}-t\right)\left(z(t)^{\prime}, z\left(t_{k}\right)^{\prime}\right) Q\left(z(t)^{\prime}, z\left(t_{k}\right)^{\prime}\right)^{\prime} .
\end{aligned}
$$

Pose $t-t_{k}=\tau, t_{k+1}-t=s$ as in our framework. Let

$$
\mathcal{Q}(\tau)=\left(\begin{array}{cc}
I_{d} & 0 \\
0 & e^{-A \tau}
\end{array}\right)^{\prime} Q\left(\begin{array}{cc}
I_{d} & 0 \\
0 & e^{-A \tau}
\end{array}\right)
$$

and

$$
M(\tau)=\int_{0}^{\tau} e^{\alpha(\rho-\tau)}\left(e^{A(\rho-\tau)}\right)^{\prime} A^{\prime} U A e^{A(\rho-\tau)} d \rho .
$$

Using straightforward computations, the functional can be expressed in the form $\bar{V}(t)=z^{\prime}(t) P(\tau(t), s(t)) z(t)$, where $P(\tau, s)=\bar{P}+s Q(\tau)+M(\tau)$, which can be related with the function $V(\xi)=z^{\prime} P(\tau, s) z$ used in our framework.

Corollary 1 Consider the hybrid system (5), (9)-(12), a matrix function $P(\tau), P: \mathbb{R} \rightarrow \mathbb{R}^{n \times n}$, with $\frac{\partial P}{\partial \tau}$ being continuous on an open set containing $[0, \bar{\tau}]$, a function $L(\tau), L: \mathbb{R} \rightarrow \mathbb{R}^{n \times q}$, continuous and bounded over $[\underline{\tau}, \bar{\tau}]$, and the following set of LMIs

$$
\begin{aligned}
& P(\tau)=P(\tau)^{\prime}>0, \forall \tau \in[0, \bar{\tau}], \\
& N_{i}(\tau)=\mathfrak{H} \mathfrak{e}\left(P(\tau) R_{i}\right)+\frac{\partial P}{\partial \tau}<0, \\
& \forall \tau \in[0, \bar{\tau}], \forall i \in \mathcal{P}, \\
& \left(\begin{array}{c|c}
\mathfrak{H} \mathfrak{e}(L(\tau) C) & C^{\prime} L^{\prime}(\tau) \\
+P(\tau)-P(0) & \\
\hline \star & P(0)
\end{array}\right)>0, \forall \tau \in[\underline{\tau}, \bar{\tau}] .
\end{aligned}
$$

If the set of LMIs (19) is verified, then the gain $K(\tau):=P(0)^{-1} L(\tau)$ ensures that the set $\mathcal{A}$ defined in (15) is UGpAS. 
PROOF. We will show that if there exists functions $P(\tau)$ and $L(\tau)$ satisfying the set of conditions (19), then the hypothesis of Theorem 2 are verified with $P(\tau, s):=P(\tau)$ and $K(\tau)=P(0)^{-1} L(\tau)$.

First note that by definition of $P(\tau):=P(\tau, s)$, the set of LMIs (19a) imply that (16a) is verified. Noting that $\frac{\partial P}{\partial s}=0$, the LMIs (19b) imply that (16b) holds.

Considering (19c) and replacing $L(\tau)$ by $P(0) K(\tau)$, one has

$$
\left(\begin{array}{c|c}
P(0) K(\tau) C & C^{\prime} K(\tau)^{\prime} P(0) \\
+P(\tau)-P(0) & \\
\hline \star & P(0)
\end{array}\right)>0
$$

holds for all $(\tau, s) \in[\underline{\tau}, \bar{\tau}]^{2}$. Therefore all the hypothesis of Theorem 2 are verified, which conclude the proof.

\subsection{Tractable conditions for synthesis}

The conditions exposed in Corollary 1 are sufficient to synthesise an observation gain for the uniform pre-asymptotic stability of the set $\mathcal{A}$, ensuring asymptotic convergence of the observer (3). However the set of LMIs (19) are infinite dimensional because of the continuous dependency in $\tau$. We will therefore give a particular instance of $P(\tau)$ that will allow for the practical computation of the observer gain.

Theorem 3 Consider the hybrid system (5), (9)-(12). If there exist positive definite matrices $P_{1}, P_{2} \in \mathbb{R}^{n \times n}$ and matrices $L_{0}, L_{1} \in \mathbb{R}^{n \times q}$ such that

$$
\begin{gathered}
M_{p}^{1}=\mathfrak{H} \mathfrak{e}\left(P_{1} R_{p}\right)+\frac{P_{2}-P_{1}}{\bar{\tau}}<0, \forall p \in \mathcal{P}, \\
M_{p}^{2}=\mathfrak{H} \mathfrak{e}\left(P_{2} R_{p}\right)+\frac{P_{2}-P_{1}}{\bar{\tau}}<0, \forall p \in \mathcal{P}, \\
\left(\begin{array}{cc}
\mathfrak{H} \mathfrak{e}\left(L_{0} C\right)-\frac{\tau\left(P_{1}-P_{2}\right)}{\bar{\tau}} & C^{\prime} L_{0}^{\prime} \\
\star & P_{1}
\end{array}\right)>0, \\
\left(\begin{array}{cc}
\mathfrak{H} \mathfrak{e}\left(L_{1} C\right)-\left(P_{1}-P_{2}\right) & C^{\prime} L_{1}^{\prime} \\
\star & P_{1}
\end{array}\right)>0,
\end{gathered}
$$

are verified, then the gain

$$
K(\tau)=P_{1}^{-1}\left(L_{0}+\frac{\tau-\underline{\tau}}{\bar{\tau}-\underline{\tau}}\left(L_{1}-L_{0}\right)\right),
$$

ensures that the set $\mathcal{A}$ defined in (15) is UGpAS.

\section{PROOF.}

$$
\text { Let } P(\tau)=P_{1}\left(1-\frac{\tau}{\bar{\tau}}\right)+P_{2} \frac{\tau}{\bar{\tau}}
$$

First let us remark that $P(\tau)$ is continuously differentiable on any open set containing $[\underline{\tau}, \bar{\tau}]$. By assumption, $P_{1}, P_{2}$ are positive symmetric, then for all $\tau \in[0, \bar{\tau}], P(\tau)>0$. Hence (19a) is verified.

For $P(\tau)$ as in $(21)$ and $N_{i}(\tau)$ as defined in (19b), the following relation holds

$$
N_{i}(\tau)=M_{i}^{1}\left(1-\frac{\tau}{\bar{\tau}}\right)+M_{i}^{2} \frac{\tau}{\bar{\tau}},
$$


where we use the definition of $M_{p}^{1}, M_{p}^{2}$ in (20a) and (20b), respectively, and the fact that $\frac{\partial P}{\partial \tau}=\frac{P_{2}-P_{1}}{\bar{\tau}}$. Therefore if (20a), (20b) hold, then (19b) is verified with $P(\tau)$ as in $(21)$.

We will show now that (20c), (20d) imply (19c) with

$$
L(\tau)=\frac{(\tau-\underline{\tau})}{(\bar{\tau}-\underline{\tau})} L_{1}+\frac{\bar{\tau}-\tau}{(\bar{\tau}-\underline{\tau})} L_{0}
$$

Consider

$$
B(\tau):=\left(\begin{array}{cc}
\mathfrak{H} \mathfrak{e}(L(\tau) C)+(P(\tau)-P(0)) & C^{\prime} L(\tau)^{\prime} \\
\star & P(0)
\end{array}\right) .
$$

One has $B(\tau)=\frac{\tau-\underline{\tau}}{\bar{\tau}-\underline{\tau}} B(\bar{\tau})+\frac{\bar{\tau}-\tau}{\bar{\tau}-\underline{\tau}} B(\underline{\tau})$. Using $(20 \mathrm{c})$ and $(20 \mathrm{~d}), B(\tau)>0, \forall \tau \in[\underline{\tau}, \bar{\tau}]$.

Therefore all the hypothesis of Corollary 1 are verified with $P(\tau)$ and $L(\tau)$ as in $(21)$ and $(22)$, respectively.

Remark 5 Given $0<\underline{\tau}<\bar{\tau}$, using the finite set of LMIs (20), it is therefore possible to check the conditions of existence of a time-varying impulsive observer of the form (3).

Remark 6 In Theorem 3 the observer gain

$$
K(\tau)=P_{1}^{-1}\left(L_{0}+\frac{\tau-\underline{\tau}}{\bar{\tau}-\underline{\tau}}\left(L_{1}-L_{0}\right)\right)
$$

is well defined only for $\bar{\tau}>\underline{\tau}$ excluding de facto the case of periodic sampling (i.e. $\bar{\tau}>\underline{\tau}$ ). However, dropping (20c) and setting $K=P_{1}^{-1} L_{1}$ one would synthesize a static impulsive gain for systems under periodic sampling.

In case the inter-sampling time is not available for measurements it can be more convenient to look for a static impulsive gain. It is the subject of the next corollary.

Corollary 2 Considering system (5),(9)-(12). Suppose the set of LMIs (20) is verified for some positive definite matrices $P_{1}, P_{2} \in \mathbb{R}^{n \times n}$ and matrices $L_{1}=L_{0} \in \mathbb{R}^{n \times q}$. Then the static gain $K(\tau)=P_{1}^{-1} L_{1}$ ensures that the set $\mathcal{A}$ defined in (15) is UGpAS.

PROOF. The proof follows directly from the application of Theorem 3.

\section{Example: Flexible joint}

Let us consider the model (1) of a flexible joint described by [18], [16], [17]:

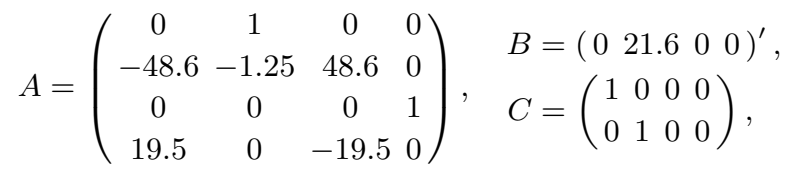

with $\phi=\left(0,0,0,3.3 \sin x_{3}\right)^{\prime}$. The input $u=\sin (t)$ is applied to the system. Using the conditions of Corollary 2 for $\underline{\tau}=0.01, \bar{\tau}=0.2$ one obtains the following gain:

$$
K=\left(\begin{array}{cccc}
0.87 & 0.03 & 0.66 & 0.65 \\
0.04 & 0.99 & 0.09 & -0.12
\end{array}\right)^{\prime}
$$


a)

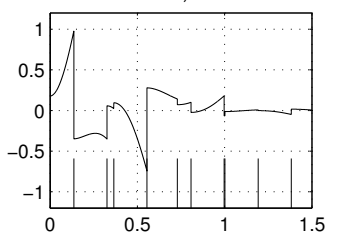

c)

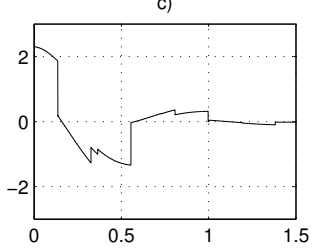

b)
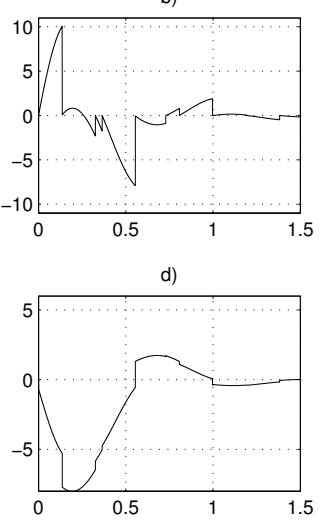

Fig. 1. Observation error of the 4 states with static gain $K$. The sampling instants are indicated in plot a), bellow the evolution of $z_{1}$.
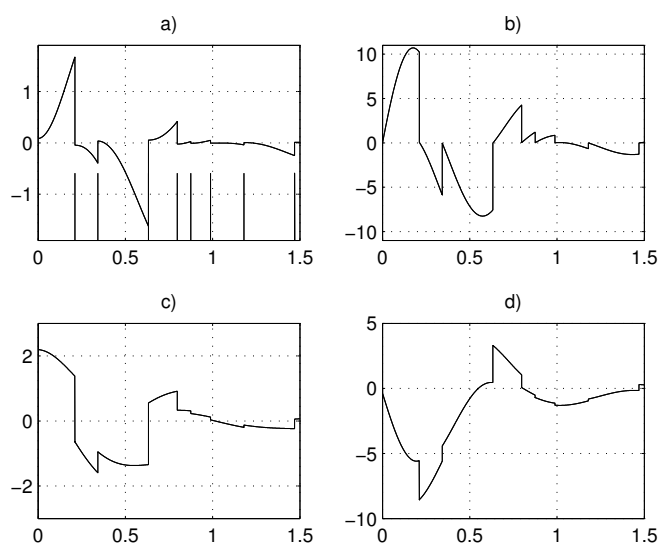

Fig. 2. Observation error of the 4 states with time-varying gain $K(\tau)$. he sampling instants are indicated in plot a), bellow the evolution of $z_{1}$.

Comparing the results of Corollary 2 with the work of [17] on the same example, Corollary 2 allows to assert convergence of the observer for a larger class of sampling sequences. Using Corollary 2 we may design an observer which is robust for all sampling sequences with $t_{k+1}-t_{k} \in[\underline{\tau}, \bar{\tau}]=[0.01,0.2]$ while in [17] the design was possible only for a sampling interval $t_{k+1}-t_{k} \in[0.01,0.1]$.

For the same example with $t_{k+1}-t_{k} \in[\underline{\tau}, \bar{\tau}]=[0.01,0.3]$, the set of LMIs in Theorem 3 allows to compute an observer gain $K(\tau)=K_{0}+\frac{\tau-\underline{\tau}}{\bar{\tau}-\underline{\tau}}\left(K_{1}-K_{0}\right)$ where

$$
\begin{gathered}
K_{0}=\left(\begin{array}{cccc}
0.92 & 0.00 & 0.77 & 0.4 \\
0.00 & 1 & 0.04 & -0.06
\end{array}\right)^{\prime}, \\
K_{1}=\left(\begin{array}{cccc}
0.98 & 0.01 & 0.91 & 0.94 \\
0.01 & 1 & 0.05 & 0.01
\end{array}\right)^{\prime},
\end{gathered}
$$

while the the BMI in [3] are not feasible. Let us remark that the class $S_{[\tau, \bar{\tau}]}$ of sampling sequences allowed by Theorem 3 is larger than the one given by Corollary 2 . 


\section{Conclusion}

In this work we have considered the problem of observation of a nonlinear Lipschitz system with discrete timevarying measurements. Observation is performed with the use of an impulsive observer. In order to synthesize an appropriate observation gain, the conditions for existence of a hybrid Lyapunov function is first established. Then, choosing a specific structure for the Lyapunov function and using convexity arguments, the proposed conditions lead to a finite set of LMIs. The resulting time-varying observer gain is a simple convex combination of two static gains. The proposed methodology encompasses the design of a static impulsive gain. The relevance of the proposed approach is illustrated by simulations performed on a robot flexible joint.

\section{References}

[1] V. Andrieu, and M. Nadri, Observer Design for Lipschitz Systems with Discrete-Time Measurements, Proceedings of the $49^{\text {th }}$ IEEE Conference on Decision and Control, pp. 6522-6527, 2010.

[2] C. Briat. Convex conditions for robust stability analysis and stabilization of linear aperiodic impulsive and sampled-data systems under dwell-time constraints. Automatica, 49(11):3449-3457, 2013.

[3] W.H. Chen, D.X. Li and X. Lu Impulsive observers with variable update intervals for Lipschitz nonlinear time-delay systems International Journal of Systems Science Vol. 44, No. 10, pp. 1934-1947, 2013.

[4] T.N. Dinh, V. Andrieu, M. Nadri, and U. Serres, Continuous-discrete-time observers design for Lipschitz systems with sampled measurements, Transactions on automatic control, pp. 787-792, 2015.

[5] M. Farza, M. M'saad, M. Fall. M.L, et al. Continuous-discrete-time observers for a class of MIMO nonlinear systems. Transactions on Automatic Control, Vol 59, No 4, pp. 1060-1065, 2014.

[6] F. Ferrante, F Gouaisbaut, R. G. Sanfelice, S. Tarbouriech. State Estimation of Linear systems in the presence of Sporadic Measurements. Automatica 2016.

[7] E. Fridman. A refined input delay approach to sampled-data control. Automatica, Vol 46, No 2, pp. 421-427, 2010.

[8] H. K. Khalil, Nonlinear Systems, Third Edition, Prentice Hall, Upper Saddle River, New Jersey, USA, 2002.

[9] C. Fiter, L. Hetel, W. Perruquetti and J.P. Richard. A robust stability framework for LTI systems with time-varying sampling. Automatica, vol. 54, no. 0, pages $56-64,2015$.

[10] R. Goebel, R.G. Sanfelice, and A.R. Teel. Hybrid Dynamical Systems: modeling, stability, and robustness. Princeton University Press, 2012.

[11] R. A. Gupta \& M. Y. Chow. Networked control system: overview and research trends. IEEE Transactions on Industrial Electronics, Vol. 57, No 7, pp 2527-2535, 2010.

[12] L. Hetel, C. Fiter, H. Omran, A. Seuret, E. Fridman, J.P. Richard and S.I. Niculescu. Recent developments on the stability of systems with aperiodic sampling: an overview. Automatica, accepted.

[13] J.P. Hespanha, P. Naghshtabrizi, and Y. Xu. A survey of recent results in networked control systems. IEEE Special Issue on Technology of Networked Control Systems, 95(1):138-162, 2007.

[14] P. Marti, R. Villa, J. M. Fuertes, \& G. Fohle. On real-time control tasks schedulability. In European Control Conference (ECC), pp. 2227-2232, 2001.

[15] F. Mazenc, V. Andrieu, and M. Malisoff, Design of Continuous-discrete Observers for time-varying nonlinear systems, Automatica, Vol. 57, pp.135-144, 2015.

[16] T. Raff and F. Allgöwer, Observer with impulsive dynamical behavior for linear and nonlinear continuous-time systems,Proceedings of the $46^{\text {th }}$ Conference Decision and Control, New Orlean, pp. 4287-4292, 2007.

[17] T. Raff, M. Kögel, and F. Allgöwer. Observer with sample-and-hold updating for Lipschitz nonlinear systems with nonuniformly sampled measurements. American Control Conference IEEE, pp. 5254-5257. 2008.

[18] M. Spong, Modeling and Control of Elastic Joint Robots, ASME Journal of Dynamic Systems, Measurement and Control, Vol. 109, pp. 310-319, 1987.

[19] W. Zhang, M.S. Branicky, and S.M. Phillips. Stability of networked control systems. IEEE Control Systems Magazine, 21(1):84-99, 2001.

[20] A. Zemouche, M Boutayeb and G.I Bara, Observer for a class of Lipschitz systems with extension to $H_{\infty}$ performance analysis, Systems and control letters, pp. 18-27 vol 57, 2008. 